

\title{
COMPORTAMIENTO TÉRMICO DE TRES PROTOTIPOS EN SALTILLO, COAHUILA (BLOQUES DE TIERRA, CONCRETO Y TAPA DE HUEVO)
}

\author{
THERMAL BEHAVIOR OF THREE PROTOTYPES IN SALTILLO, \\ COAHUILA (WITH EARTH BLOCKS, CONCRETE AND EGG CARTONS)
}

\author{
MARÍA EUGENIA MOLAR OROZCO \\ Doctora en investigación en energía y medio ambiente \\ de la Arquitectura \\ Docente e Investigadora de la Facultad de Arquitectura, \\ Universidad Autónoma de Coahuila, Unidad Saltillo, Arteaga, México \\ https://orcid.org/0000-0001-5357-5893 \\ mariamolar@uadec.edu.mx
}

\author{
JESÚS VELÁZOUEZ-LOZANO \\ Magíster en Diseño Arquitectonico \\ Docente e Investigador de la Facultad de Arquitectura, \\ Universidad Autónoma de Coahuila, Unidad Saltillo, Arteaga, México \\ https://orcid.org/0000-0003-2783-9319 \\ jvelazqu@uadec.edu.mx
}

\author{
MARÍA GENOVEVA VÁZQUEZ-JIMÁNEZ \\ Magíster en Diseño Arquitectonico \\ Docente e Investigadora de la Facultad de Arquitectura \\ Universidad Autónoma de Coahuila, Unidad Saltillo, Arteaga, México \\ https://orcid.org/0000-0002-5150-9726 \\ gvazquez@uadec.edu.mx
}

\begin{abstract}
RESUMEN
El cambio climático se ha ido agravando en el siglo XX, genera modificaciones estacionales a nivel global en el hábitat, se observan variaciones importantes en los factores climáticos, aumentando las temperaturas en las ciudades. En México el acondicionamiento térmico de las viviendas repercute en gran medida sobre la demanda de electricidad, siendo mayor en las zonas norte y costeras del país, el rol de la envolvente es mantener un equilibrio entre el exterior y el interior, refiriéndose a las ganancias o pérdidas de calor, que se logran a través de su transferencia producto de las variaciones de la temperatura. El objetivo del trabajo fue comparar el comportamiento térmico de tres módulos ubicados en Saltillo, Coahuila; construidos de tres distintos materiales: uno experimental y dos de

uso común en las viviendas de Saltillo, la metodología es de enfoque cuantitativo, se realizaron mediciones del 2018 al 2019, los resultados muestran que el material experimental con respecto a los otros materiales comerciales no tienen diferencias relevantes en los meses más críticos que son enero y mayo, apenas 1 o 2 grados, por lo que es pertinente seguir experimentando y complementar con un sistema pasivo, como un pozo canadiense, con la meta de mejorar el confort en el hábitat construido.
\end{abstract}

\author{
Palabras clave \\ Bloque, edificación, temperatura ambiental
}

\begin{abstract}
Climate change has been worsening in the 20th century, causing seasonal changes in the habitat at a global level. Significant variations in climate factors are seen, increasing temperatures in cities. In Mexico, thermal conditioning of houses has a major impact on the electricity demand, which is greater in the northern and coastal areas of the country. The role of the envelope, when referring to heat gains or losses that are achieved by their temperature variation transfer, is to maintain a balance between the outside and the inside. The goal of this work was to compare the thermal behavior of three modules located in Saltillo, Coahuila; built using three different materials, one experimental and two commonly used in Saltillo homes. The methodology has a quantitative focus and measurements were made from 2018 to 2019. The results show that there are no relevant differences between the experimental material and other commercial materials in the most critical months, January and May, with just 1 or 2 degrees.

Thus, it is relevant to continue experimenting and complementing with a passive system, like a Canadian well, with the goal of improving comfort in the built habitat.
\end{abstract}




\section{INTRODUCCIÓN}

El cambio climático en el siglo $\mathrm{XX}$ ha generado modificaciones estacionales a nivel global en el hábitat, observándose variaciones importantes en los factores del clima que afectan directamente el confort al interior de las viviendas. En efecto, los climas que antes se consideraban templados ya no mantienen esta categoría, en tanto que, los climas cálidos son más extremos que antes, lo que trae como consecuencia la necesidad de adaptar el hábitat de las viviendas para mejorar el confort.

A juicio de Delfín, Gallina y López (2014), el hábitat tiene la responsabilidad de cumplir con las condiciones adecuadas para una especie, partiendo de dos vertientes: hábitat real, que se refiere a la presencia de la especie en un espacio, y hábitat potencial, que implica que un área donde no está presente una especie potencialmente pueda constituir hábitat para ella.

La Organización Panamericana de la Salud (OPS, 1999) señala que, la estructura de la vivienda para grupos en pobreza no presenta las condiciones necesarias para servir como albergue que proteja adecuadamente de temperaturas extremas, ruido, entre otros factores. Múnera (2011), por su parte, considera que el hábitat y, en particular, la vivienda, se convierten en "objetos" de intervención y manipulación, convirtiéndose en mercancía, al estandarizar la producción en serie de la vivienda de interés social por parte del sector privado.

La Secretaría de Energía (SENER y CONUEE, 2011) indica que:

En México, el acondicionamiento térmico de las viviendas repercute en gran medida en la demanda pico del sistema eléctrico, siendo mayor su impacto en las zonas Norte y costeras del país, en donde es más común el uso de sistemas activos. (p.1)

En este marco, Herrera (2017) señala que se establecen especificaciones técnicas de aplicación obligatoria, entre ellas, la Norma Mexicana Eficiencia Energética en Edificaciones y la Envolvente de edificios para uso habitacional (NOM-020-ENER-2011), cuyo objetivo es el de:

Limitar la ganancia de calor de los edificios para uso habitacional a través de su envolvente, racionalizando el uso de la energía en los sistemas de enfriamiento y mejorando las condiciones de confort térmico al interior de los espacios de la vivienda. (SENER y CONUEE, 2011, p. 1)

García, Kochova, Pugliese y Sopoliga (2010) plantean que una vivienda es como una caja que respira, ya que en base al clima externo activa diferentes mecanismos para regular el calor; pero, además, una construcción depende del diseño, forma y envolvente, dando como resultado confort o disconfort por dos parámetros constantes: la temperatura y la humedad. Ambos juegan un rol importante en el resultado final. Estos autores junto con Costantini, Carro Pérez y Francisca (2016) sugieren que:

La elección de los materiales de construcción es clave para alcanzar altos niveles de confort a un bajo coste. Por ejemplo, un ladrillo cerámico hueco tiene muy buenas propiedades aislantes (o alta resistencia térmica) pero existen otros materiales como la arcilla térmica que aun presenta un mejor rendimiento (p.12).

La Comisión Nacional de Vivienda (CONAVI) conjuntamente con la Agencia Alemana de Cooperación Técnica (GIZ) implementaron el programa de Medidas de mitigación apropiadas a cada país (NAMA) para desarrollar vivienda sustentable en México. El problema es que existen escasos materiales con etiqueta verde, con base en la capacidad térmica y el retardo térmico. Este último se refiere al tiempo en que pasa el calor o frío del exterior al interior (Morris, 2017) para mantener confortable un espacio sin la necesidad de utilizar un sistema artificial $y$, por consiguiente, sin generar una alta demanda de consumo de energía (Roux, 2018).

De acuerdo con Calderón (2019), es posible construir un hábitat sustentable usando materiales reciclados y de precios bajos, sin afectar el presupuesto destinado para su construcción y, a su vez, mejorar el confort térmico. Asimismo, Herrera (2017) afirma que utilizar de manera adecuada los materiales de construcción de acuerdo con sus propiedades térmicas permite que las viviendas se acerquen a niveles de confort en cada una de las zonas climáticas, impactando menos al entorno próximo y demandando menos energía no renovable. Ante todo, recomienda evaluar los espesores de masa térmica, incluso las dimensiones de los materiales estudiados.

La envolvente tiene la función de mantener un equilibrio entre el exterior y el interior, refiriéndose a las ganancias o pérdidas de calor que se logran a través de su transferencia producto de las variaciones de la temperatura exterior. En invierno, el calor se genera en el interior de la construcción y se pierde en espacios con baja temperatura o se dispersa al exterior por las aberturas; en verano, la ganancia se obtiene del exterior al interior, debido a la falta de protección, o bien, de los materiales que son buenos conductores y de las condiciones del entorno que no ayudan a reducir el aumento de energía en el espacio interior.

Por otra parte, con el fin de alcanzar temperaturas interiores de bienestar térmico, en todo tipo de edificio, la hermeticidad juega un rol importante para contribuir a una reducción o aumento de la temperatura interior (Molina, Lefebvre, Horn y Gómez, 2020). Muñoz, Marino y Thomas (2015) consideran la orientación de una construcción un factor sobre el consumo energético necesario para su funcionamiento, por lo que al evaluar su comportamiento se debe tomar en cuenta los aportes de los componentes de la envolvente (muros, aberturas y techos). 
En estudios previos realizados por Molar, Velázquez y Gómez (2018), se señala que:

\begin{abstract}
En mayo las temperaturas de las viviendas muestran un comportamiento térmico acorde a los rangos de confort en verano, pero en enero se tienen temperaturas muy bajas, con una gran amplitud térmica entre el día y la noche, en consecuencia, se demanda el uso de calefacción para mejorar las condiciones al interior ( $p$. 7).
\end{abstract}

La lectura de temperatura interior fuera de estos rangos manifiesta que las pérdidas o ganancias de calor son producto de una inadecuada selección de los materiales de la envolvente. Si bien, en ocasiones, esto se debe a aberturas, determinada orientación y materiales en general, se ha explorado que por unidad de área son los elementos de la envolvente los que más trasfieren calor del exterior al interior (Huelsz, Molar y Velázquez, 2014; Espinoza, Cordero, Ruíz y Roux, 2017). El proceso de transferencia de calor se produce por la captación de la radiación solar conducida a través del material hacia el interior y liberada gracias a la convección, lo cual afecta el comportamiento térmico ambiental al interior del edificio.

En pruebas de simulación con el programa Ener-habitat para el clima de Saltillo (Molar y Huelsz, 2017), se comparó el valor de la carga térmica total del mes de mayo, según un espesor determinado. De entre distintas orientaciones, la que registró mayor carga fue la oeste; pero, en enero, e iguales condiciones, la orientación con mayor carga fue la norte.

Otro aspecto recomendado es el de analizar una edificación de forma anual (Rodríguez, Nájera y Martín, 2018), lo que significa que, si solo se estudian las condiciones de verano o invierno, es posible mejorar el desempeño térmico en un solo periodo, pero podría afectarse el otro, de lo que resultaría la neutralización de las ganancias o ahorros.

En virtud de lo anterior, el CA de Tecnología en la Arquitectura de la Facultad de Arquitectura de la Universidad Autónoma de Coahuila Campus Arteaga, ha realizado investigación con materiales de desechos industriales no tóxicos y naturales, con el objetivo de desarrollar sistemas constructivos sustentables que mejoren las condiciones de confort térmico del hábitat construido. En este artículo se presentan, precisamente, los resultados de un proyecto llevado a cabo en ese contexto y desarrollado desde el año 2018 al 2019. En él se compara el comportamiento térmico de la envolvente de tres módulos ubicados en Saltillo, Coahuila, construidos con tres distintos materiales: el bloque de concreto (el más empleado en la construcción), el bloque de tierra comprimida (típico de localidad) y un material experimental previamente probado como sistema constructivo (Velázquez y Molar, 2016). El objetivo es conocer sus resultados de acuerdo a orientaciones determinadas.

\section{METODOLOGÍA}

El enfoque de la investigación es cuantitativo, de forma transversal, con un trabajo documental y estudio de caso en campo. Concretamente, se hicieron mediciones en el sitio, de acuerdo con la norma ASTM Standard Practice for In-Situ Measurement of Heat Flux and Temperature on Building Envelope Components, la cual establece que se debe recoger información del entorno del hábitat analizado y luego compararla con los datos obtenidos al interior de los 3 módulos.

Así, se emplearon dos dispositivos:

1. Para medir la temperatura de las superficies, se empleó un termómetro infrarrojo, al cual se introduce el tipo de emisividad de acuerdo con el material de la envolvente horizontal y vertical, siempre cuidando medir en el mismo punto, a una altura intermedia de la superficie. Las tomas de temperatura se realizaron en la parte central de los muros al interior y exterior; en el caso de la cubierta solo se registró la parte interior, en el centro de la superficie.

2. Para medir la temperatura ambiente y el porcentaje de humedad, se emplearon dos registradores datalogger de temperatura y humedad relativa: uno para el exterior y el otro para el interior de cada módulo. Las mediciones se realizaron bajo sombra en el exterior y al interior en la parte intermedia del espacio.

Durante el periodo de medición, los módulos se mantuvieron en las mismas condiciones, no fueron habitados por nadie, por lo tanto, no hubo ganancia interna, pero sí se consideró ganancia solar por conducción y convección por los vanos.

Las mediciones se efectuaron de forma periódica, una semana al mes, en las jornadas próximas al día de solsticios y equinoccios, a cada hora, entre las 9:00 a las 15:00 horas, rango de mayor captación de radiación, a partir de mayo de 2018 hasta mayo de 2019. Se compararon, posteriormente, los datos exteriores con los interiores de los hábitats, registrando solo los datos diurnos, debido a que las condiciones de seguridad no permitían realizar mediciones nocturnas. Los datos se vaciaron en un formato y fueron procesados; se identificaron entonces los meses más críticos, descartándose los demás por ubicarse dentro de la zona de confort. En este caso, solo se muestran los resultados de mayo y enero.

Con propósitos comparativos, se determinó la zona de confort para Saltillo, Coahuila, de acuerdo con los criterios de Szokolay (2014) y ANSI/ASHRAE Standard 55-2010 que proporciona el programa CONFORT ANSI ASHRAE de Excel de Luna (2019), para verano e invierno.

Para el cálculo de la temperatura neutra, se emplea la fórmula de Auliciems (Szokolay, 2014, p. 20) (ecuación 1). 


$$
T n=17.6+0.31 \mathrm{Tmm}
$$

Donde Tn es la temperatura neutra y Tmm es la temperatura promedio del mes.

$\operatorname{Tn}=17.6+(0.31 * 12.1)$

$\mathrm{Tn}=21.4^{\circ} \mathrm{C}$ para el mes de enero y

$\mathrm{Tn}=17.6+(0.31 * 22.3)$

$\mathrm{Tn}=24.5^{\circ} \mathrm{C}$ para el mes de mayo

Los rangos de temperatura de confort oscilan entre ( $\mathrm{Tn}$ 2.5) ${ }^{\circ} \mathrm{C}$ a $(\mathrm{Tn}+2.5){ }^{\circ} \mathrm{C}$, según lo plantea Szokolay (2014, p. 21), por lo que, para Saltillo, en el mes de enero, cuya temperatura promedio es de $12.1^{\circ} \mathrm{C}$, el rango de confort es de 21.4 a $23.9^{\circ} \mathrm{C}$; mientras que en el mes de mayo, con temperatura promedio de $22.3^{\circ} \mathrm{C}$, los valores de confort van de 22 a $27^{\circ} \mathrm{C}$.

\section{DESCRIPCIÓN DE LOS PROTOTIPOS}

El proyecto se realizó en las instalaciones de la Universidad Autónoma de Coahuila, los tres módulos están ubicados dentro de la zona universitaria denominada Camporredondo, en Saltillo, Coahuila (Figura 1).

La ciudad de Saltillo se encuentra a 1600 metros sobre el nivel del mar, con latitud $25^{\circ} 22^{\prime} 35^{\prime \prime}$ y longitud $101^{\circ} 01^{\prime} 00^{\prime \prime}$, según CONABIO (Comisión Nacional para el Conocimiento y Uso de la Biodiversidad), posee un clima seco templado y seco cálido con pocas precipitaciones a lo largo del año.

Los tres hábitats (Tabla 1) cuentan con similar dimensión en largo, alto, ancho (por el tamaño de los bloques) y misma orientación (NE, ES, SO y NO), se procuró cierta separación para no generar sombra entre sí, ni obstruir la circulación del aire. La envolvente (muros y cubierta) de cada módulo corresponde a cada tipo de material: bloque de tierra comprimida (BTC), bloque de concreto $(\mathrm{BC})$ y bloque de tapa de huevo con termolita y cemento (BTH) (Tabla 2). Ninguno de los módulos cuenta con recubrimiento en exterior ni interior; sí, con dos aberturas, una pequeña ventana y la puerta, en orientación SO (Figura 2).

\begin{tabular}{|c|c|}
\hline \multirow[t]{2}{*}{ Módulo } & Dimensión m \\
\hline & (ancho, largo y alto) \\
\hline $\begin{array}{l}\text { Módulo de Bloque de tierra } \\
\text { comprimida (BTC) }\end{array}$ & $1.4 \times 2.20 \times 2.36$ \\
\hline $\begin{array}{l}\text { Módulo de Bloque de concreto } \\
\text { (BC) }\end{array}$ & $1.47 \times 2.26 \times 2.50$ \\
\hline $\begin{array}{l}\text { Módulo de Bloque de tapa de } \\
\text { huevo con termolita y cemento } \\
\text { (BTH) }\end{array}$ & $1.57 \times 2.21 \times 2.45$ \\
\hline
\end{tabular}

Tabla 1. Dimensión de módulos.

Fuente: Elaboración de los autores.
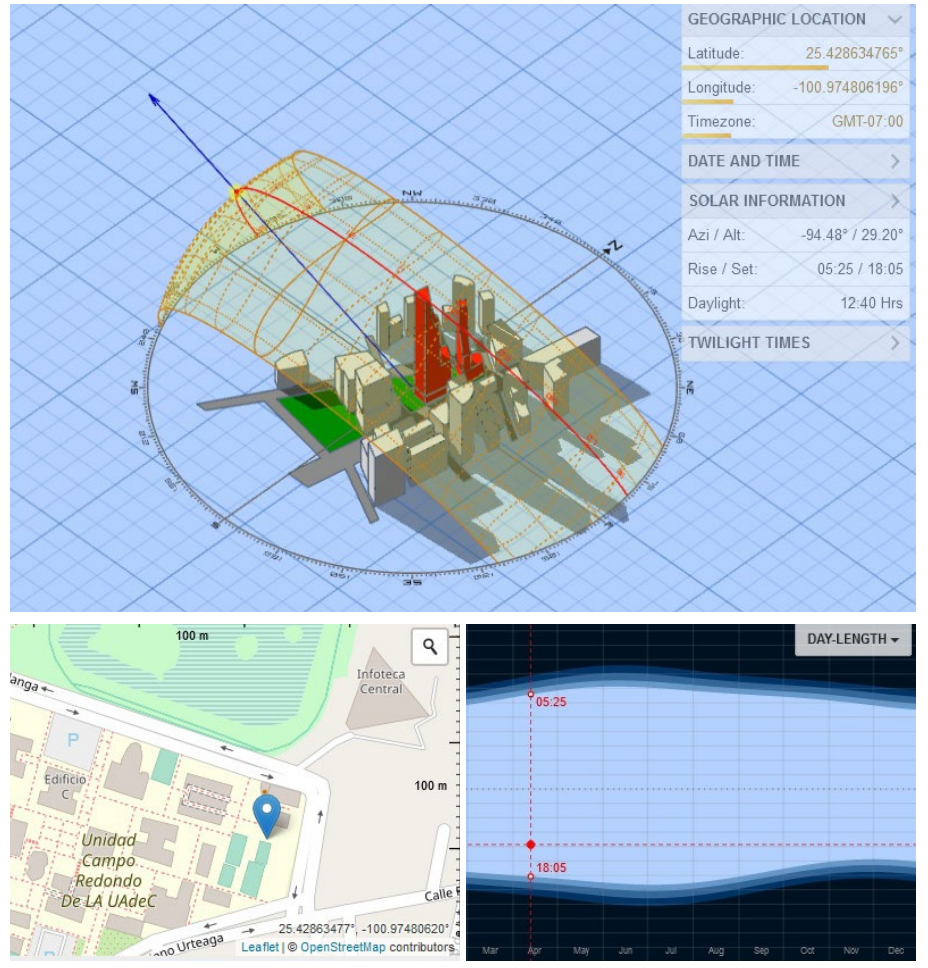

Figura 1. Ubicación de los módulos. Fuente: Andrewmarsh.com. PD: 3D Sun Path.

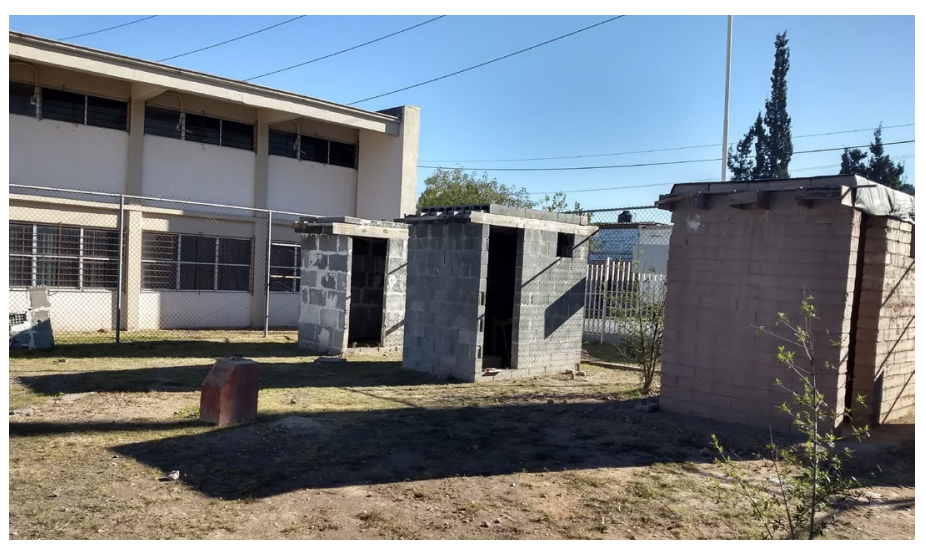

Figura 2. Vista de los módulos (de izquierda a derecha: de bloques de tapa de huevo con termolita y cemento -BTH-, de bloque concreto - BCy de bloque de tierra comprimida -BTC-). Fuente: Elaboración del autor.

\begin{tabular}{|c|c|}
\hline Material & $\begin{array}{c}\text { Dimensión cm } \\
\text { (ancho, largo y alto) }\end{array}$ \\
\hline $\begin{array}{c}\text { Bloque de tierra comprimida } \\
\text { (BTC) }\end{array}$ & $20 \times 40 \times 12$ \\
\hline Bloque de concreto & $14.5 \times 39.5 \times 19$ \\
\hline $\begin{array}{c}\text { Bloque de tapa de huevo con } \\
\text { termolita y cemento (BTH) }\end{array}$ & $10 \times 69 \times 35$ \\
\hline
\end{tabular}

Tabla 2. Dimensiones de los bloques.

Fuente: Elaboración de los autores. 
El prototipo experimental BTH, compuesto por materiales ligeros como termolita, cemento portland, arena y tapa de huevo, fue probado en 2015 por el Dr. Raúl Ernesto Canto Cetina y el Dr. Porfirio Nanco Hernández, obteniéndose una Conductancia de $2.59 \mathrm{~W} / \mathrm{m}^{2}{ }^{\circ} \mathrm{C}$, con una Resistencia térmica de $386 \mathrm{~m} 2^{\circ} \mathrm{C} / \mathrm{W}$ (Velázquez y Molar, 2016).

\section{RESULTADOS Y DISCUSIÓN}

Los Gráficos 1 a 5 corresponden a los datos obtenidos en enero de 2019, a partir de los cuales se advierte que la máxima temperatura se alcanza entre las 12:00 y 13:00 h en la orientación SO. En general, la temperatura de la superficie al interior registra alrededor de 15 a $10^{\circ} \mathrm{C}$ de diferencia respecto a la superficie exterior, aun con los vanos abiertos.

La máxima temperatura en las superficies exteriores en el módulo de bloque de tierra comprimida (BTC) (Gráfico 1) se da en la orientación SE y SO a las $12: 00$ y $13: 00$ h, alcanzando 34 y $35^{\circ} \mathrm{C}$, respectivamente, esto es, $15^{\circ} \mathrm{C}$ de diferencia respecto a las superficies interiores; mientras que las lecturas de las orientaciones NE y NO presentan un comportamiento similar de las caras interiores, al no tener radiación directa. A las 14:00 h se observa que, aun cuando la superficie exterior NO inicia el ascenso de temperatura al recibir radiación, su cara interior no aumenta la temperatura, presentándose una diferencia entre ellas de $4^{\circ} \mathrm{C}$. A las 11:00, el aumento de temperatura de las superficies interiores fue de $5^{\circ} \mathrm{C}$ y a las $13: 00$, volvieron a descender.

La máxima temperatura en la superficie exterior en el módulo de muro de bloque de concreto (BC) (Gráfico 2) se da en la orientación $\mathrm{SO}$ a las $13: 00 \mathrm{~h}$, con $43^{\circ} \mathrm{C}$, es decir, $20^{\circ} \mathrm{C}$ de diferencia respecto a su superficie interior. En este módulo las temperaturas de las caras interiores de las superficies a las 11:00 h tuvieron un aumento de 5 a $10^{\circ} \mathrm{C}$ de diferencia con respecto a la temperatura ambiente exterior. Las superficies exteriores NO y NE mostraron de 5 a $8^{\circ} \mathrm{C}$ más que la temperatura exterior, aun estando bajo sombra, alcanzando $25^{\circ} \mathrm{C}$.

La temperatura del módulo construido con bloques de tapa de huevo con termolita y cemento (BTH) (Gráfica 3) evidencia que la máxima temperatura en la superficie exterior se genera en la orientación SO a las 13:00 horas, con $45^{\circ} \mathrm{C}$ y $20^{\circ} \mathrm{C}$ de diferencia respecto a la cara interior, las temperaturas interiores de las superficies se mantuvieron por arriba de la temperatura ambiente exterior, entre 5 y $7^{\circ} \mathrm{C}$ de diferencia. Mientras, las superficies exteriores NO y $\mathrm{NE}$ registraron de 5 a $9^{\circ} \mathrm{C}$ de distancia, aun cuando no hubo radiación directa; pero la temperatura al interior de estas superficies se mantuvo $3^{\circ} \mathrm{C}$ por debajo.

De acuerdo con el Gráfico 4, en esta temporada no se alcanzan altas temperaturas en las cubiertas debido al contacto del aire frío que tiende a absorber el calor de

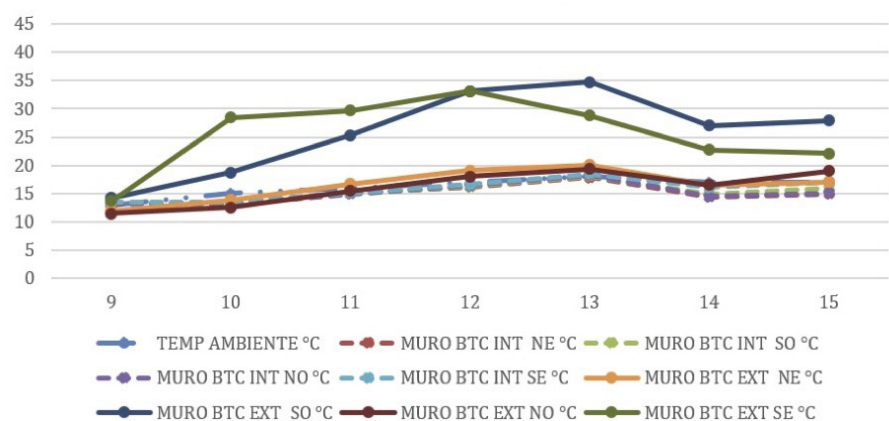

Gráfico 1: Comportamiento de temperaturas exteriores e interiores de muros del módulo de BTC (enero 2019). Fuente: Elaboración de los autores.

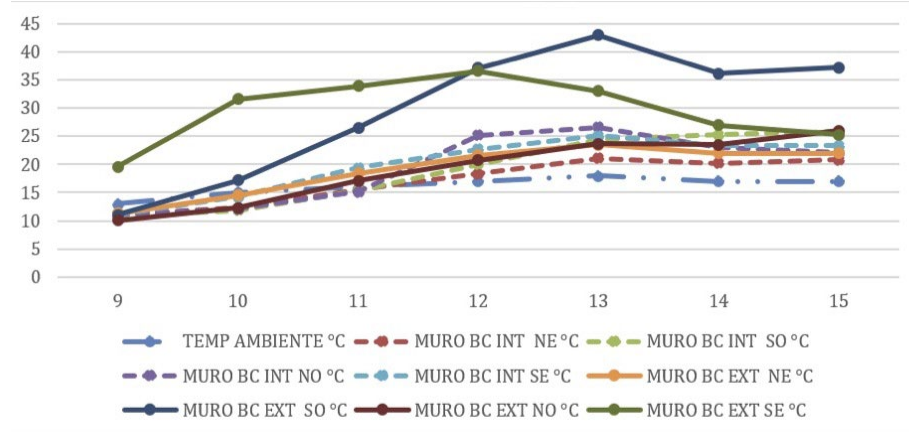

Gráfico 2. Comportamiento de temperaturas exteriores e interiores de muros del módulo de BC (enero 2019). Fuente: Elaboración de los autores.

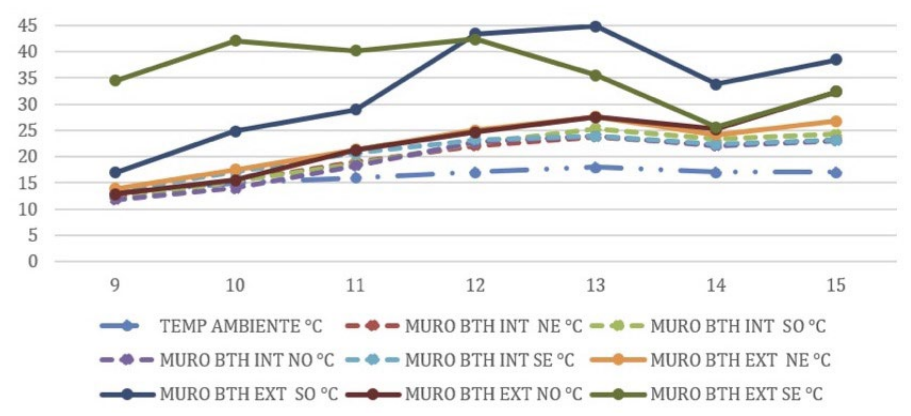

Gráfico 3. Comportamiento de temperaturas exteriores e interiores de muros del módulo de BTH (enero 2019). Fuente: Elaboración de los autores.

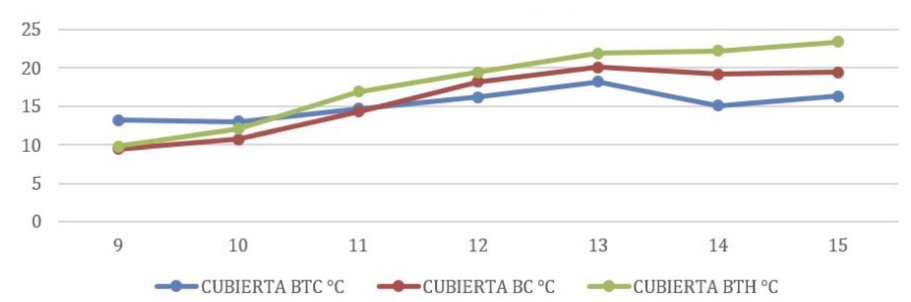

Gráfico 4. Comportamiento de las temperaturas de la superficie interior de la cubierta de los tres módulos (BTC, BC y BTH), enero 2019. Fuente: Elaboración de los autores. 
las superficies expuestas. La temperatura máxima fue alcanzada por la cubierta con BTH y fue de $24^{\circ} \mathrm{C}$; la más baja, se consiguió con la cubierta de BTC, y fue de $18^{\circ} \mathrm{C}$. El $\mathrm{BC}$ y $\mathrm{BTH}$ inician con una misma temperatura al principio del registro.

ANSI/ASHRAE 55:2010

\begin{tabular}{|c|c|c|}
\hline \multicolumn{3}{|c|}{ ENERO } \\
\hline \multicolumn{3}{|c|}{ 80\% ACEPTACION } \\
\hline L. INF. & TN & L. SUP. \\
\hline 19.50 & 22.00 & 24.50 \\
\hline
\end{tabular}

Figura 3. Límites de confort en enero. Fuente: Programa CONFORT ANSI ASHRAE.

Con base en el límite de confort correspondiente a enero, según Szokolay, $21.4^{\circ}$ a $23.9^{\circ} \mathrm{C}$, y el obtenido con el programa CONFORT ANSI ASHRAE, se dispusieron los límites en el diagrama para proceder a realizar análisis de los resultados obtenidos de las mediciones.

Aun cuando las envolventes de los tres módulos (Gráfico 5) tienen variaciones en las temperaturas de las superficies, el comportamiento resultante en los tres módulos fue similar con una temperatura ambiente, alcanzando el límite inferior (Figura 3). A partir de las 11 horas, capturan energía a través de la envolvente, aunque en el caso del BTH se obtuvo valores ligeramente más altos y en el de BTC se registró valores más bajos.

Los Gráficos 6 a 8 pertenecen a los datos de mayo 2019 (figura 4). La máxima temperatura de las superficies exteriores se da en la orientación SE, a las 11:00.

En el módulo de muro de block de tierra comprimida (Gráfico 6), la máxima temperatura de la superficie exterior se registró en la orientación SE a las $11: 00 \mathrm{~h}$, con $56^{\circ} \mathrm{C}$ y $25^{\circ} \mathrm{C}$ de diferencia respecto a la superficie interior; la temperatura de las superficies interiores aumentó de forma gradual a las 10:00 h, pero se mantuvo por debajo de la temperatura ambiente.

En el módulo de BC (Gráfico 7), la máxima temperatura de las superficies exteriores se da en la orientación SE a las $11: 00 \mathrm{~h}$, con $56^{\circ} \mathrm{C}$ y $20^{\circ} \mathrm{C}$ de diferencia respecto a la superficie interior; la temperatura interior de las superficies se incrementó a las 10:00 h, presentando una diferencia con la temperatura exterior de entre 1 y $8^{\circ} \mathrm{C}$. A las 11:00 h, las superficies exteriores $\mathrm{NO}$ y $\mathrm{SO}$ aumentaron su temperatura de forma gradual, exhibiendo una diferencia de entre 8 y $10^{\circ} \mathrm{C}$ respecto a la temperatura exterior, con una oscilación de $5^{\circ} \mathrm{C}$ respecto a la superficie interior.

En el módulo de BTH (Gráfico 8), la máxima temperatura de las superficies exteriores se produce en la orientación SE a

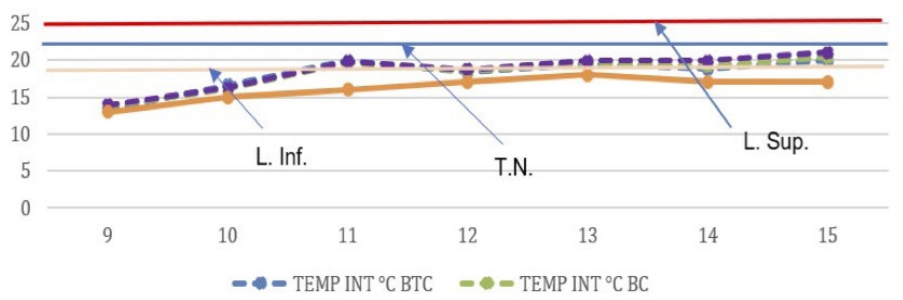

Gráfico 5. Comportamiento térmico interior de los tres módulos (enero 2019).Fuente: Elaboración de los autores.

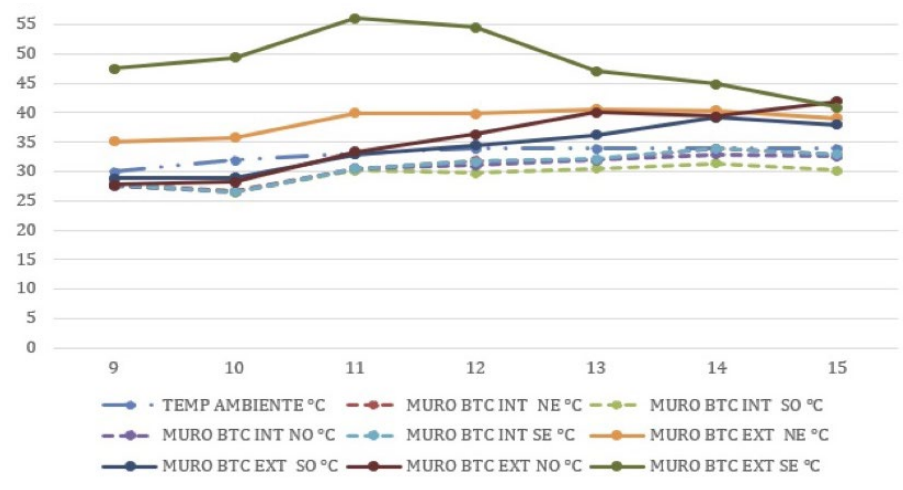

Gráfico 6. Comportamiento de temperaturas exteriores e interiores de muros del módulo de BTC (mayo 2019). Fuente: Elaboración de los autores.

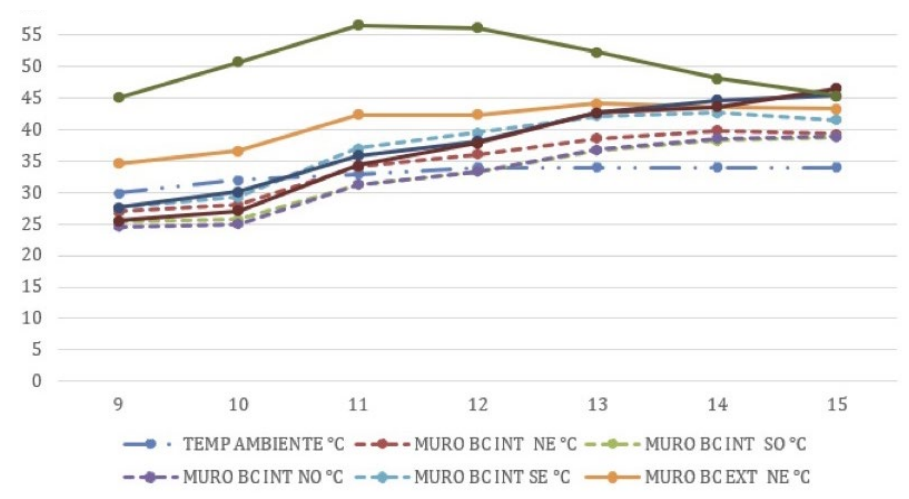

Gráfico 7. Comportamiento de temperaturas exteriores e interiores de muros del módulo de BTC (mayo 2019). Fuente: Elaboración de los autores.

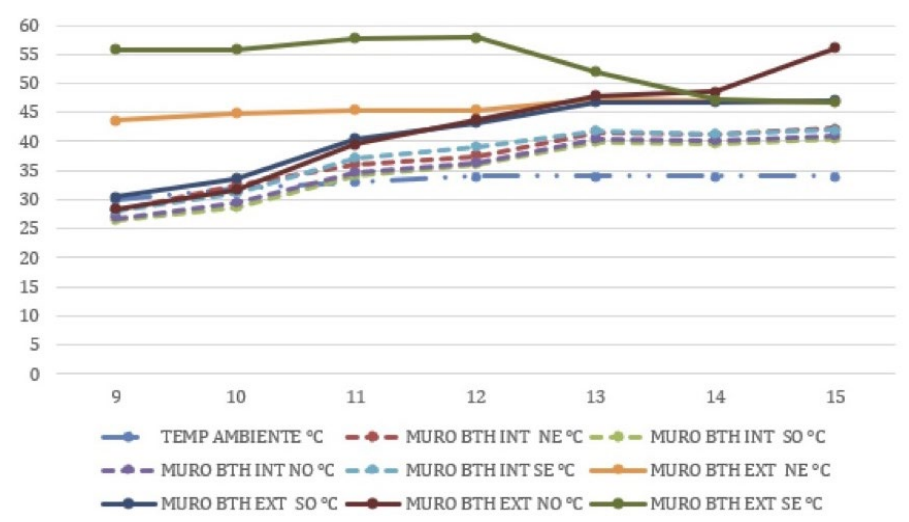

Gráfico 8. Comportamiento de temperaturas exteriores e interiores de muros del módulo de BTH (mayo 2019).Fuente: Elaboración de los autores. 
las $11: 00$ y $12: 00 \mathrm{~h}$, con $57^{\circ} \mathrm{C}$ y $18^{\circ} \mathrm{C}$ de diferencia respecto a la superficie interior. La temperatura interior de las superficies se incrementó a partir de las 10:00 h, registrando una diferencia 1 a $7^{\circ} \mathrm{C}$ respecto a la temperatura exterior. Se observó, asimismo, que las superficies exteriores NO y SO empezaron a aumentar a 10:00 h, manteniendo una diferencia de 5 a $20^{\circ} \mathrm{C}$ respecto a la temperatura exterior, con una oscilación de $5^{\circ} \mathrm{C}$ con su respectiva superficie interior.

En el Gráfico 9, se aprecia que la temperatura máxima se presenta en la cubierta de BTH y es $43^{\circ} \mathrm{C}$; y la más baja, en la de BTC, de $36^{\circ} \mathrm{C}$. En este periodo las tres cubiertas inician con temperaturas similares a las 9:00 $\mathrm{h}$, en tanto que a las 15:00 h mantienen una diferencia de $7^{\circ} \mathrm{C}$ entre el valor menor y el mayor

\begin{tabular}{|c|c|c|}
\hline \multicolumn{3}{|c|}{ MAYO } \\
\hline \multicolumn{3}{|c|}{ 80\% ACEPTACION } \\
\hline L. INF. & TN & L. SUP. \\
\hline 22.70 & 25.20 & 27.70 \\
\hline
\end{tabular}

Figura 4: Límites de confort en mayo. Fuente: Programa CONFORT ANSI ASHRAE.

Con base en el límite de confort correspondiente a mayo, según Szokolay, $22^{\circ}$ a $27^{\circ} \mathrm{C}$, y el obtenido con el programa CONFORT ANSI ASHRAE, se definieron los límites en el diagrama para proceder al análisis de los resultados obtenidos por las mediciones.

La temperatura interior resultante de los tres módulos (Gráfico 10) tiene un comportamiento térmico similar, aunque a las 12:00 h las temperaturas del BTH y BC son ligeramente más altas que la del BTC. Los tres se encuentran fuera del límite superior, manteniendo una temperatura inferior a la exterior en las primeras horas.

Según lo señalado por García et al. (2010), una envolvente activa sus mecanismos de regulación de acuerdo con las propiedades del material y su intercambio con el exterior. Un aspecto clave, como plantean Huelsz et al. (2014), Espinoza et al., (2017) y Muñoz et al. (2015) es la afectación generada por las aberturas que influyen en gran medida en la ganancia o pérdida de transferencia de calor al interior de la construcción. La hermeticidad, es la clave para reducir o aumentar la temperatura interior (Molina et al., 2020).

\section{CONCLUSIÓN}

Al comparar el comportamiento térmico en invierno de los tres módulos localizados en Saltillo, Coahuila, a partir de las orientaciones NE, ES, SO y NO de los muros, se

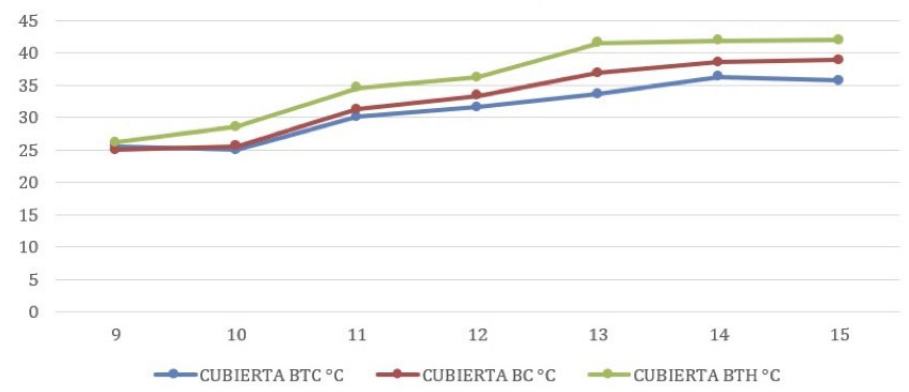

Gráfico 9. Comportamiento de las temperaturas de la superficie interior de la cubierta de los tres módulos (BTC, BC y BTH) (mayo 2019).

Fuente: Elaboración de los autores.

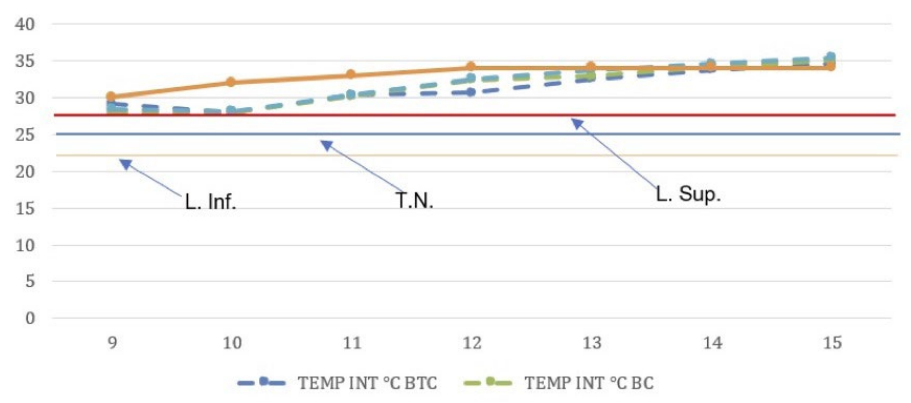

Gráfico 10. Comportamiento térmico interior de los tres módulos (mayo 2019).Fuente: Elaboración de los autores.

identifica que, en invierno, la suroeste mantiene los valores más altos en el transcurso de la mañana - tarde, debido a que recibe mayor cantidad de radiación solar a esa hora del día. Asimismo, dada la composición de los bloques y a partir de las distintas orientaciones, la sureste, en verano, tiene mayor energía transmitida en el transcurso de la mañana - tarde, entre las 11:00 y las 13:00 h, periodo en que las superficies captan la mayor cantidad de radiación. Esto constituye un área de oportunidad a considerar, puesto que en invierno se requiere aprovechar el calor y en verano se pretende tener menos captación, lo cual permitiría proponer alguna alternativa en el diseño en ambas orientaciones.

Comparando las temperaturas interiores ambientales del mes de enero de los tres módulos con los rangos de confort, a partir de Szokolay (2014)y del programa de Luna (2019), las lecturas inician con valores por debajo de los rangos de confort, aunque a las 15:00 se ubicaron dentro del rango de confort. Por otra parte, en mayo, los tres módulos estuvieron por arriba de los rangos de confort, lo cual significa que el mayor problema a trabajar surgiría en verano.

Respecto a la comparativa entre las superficies de los materiales experimentales (BTC y BTH) y el bloque de concreto comercial (BC), estos muestran diferencias relevantes en enero y mayo, por lo que es posible que el espesor del bloque experimental sea muy delgado 
para contribuir en la mejora de las condiciones al interior. Aun así, es importante seguir trabajando en nuevas alternativas y mejoras del material para implementarlo en construcciones de viviendas de bajos recursos y mejorar el hábitat construido.

La envolvente con el BTC en ambas estaciones mantiene siempre temperaturas por debajo o similares a la temperatura ambiente, en cambio, los BC y BTH tienden a aumentar la temperatura de forma gradual a partir de las 10:00 y 11:00 $\mathrm{h}$ respecto a la temperatura exterior; no obstante, el resultado final fue similar a la temperatura interior en los tres materiales. Cabe señalar que el comportamiento de las superficies en sus caras interiores fue diverso, lo cual ofrece la opción de seguir trabajando en mejoras.

Estos resultados pueden estar asociados a la falta de protección en los vanos, al espesor de los bloques tanto en muros como en cubierta, o bien, a la falta de algún empastado exterior e interior que reduzca el incremento de la temperatura ambiental al interior de la construcción. Por lo que es pertinente seguir realizando pruebas con otras geometrías en los bloques experimentales, cerrar las aberturas y complementar con un pozo canadiense como sistema pasivo auxiliar que, de acuerdo con cálculos matemáticos de Molar, Ríos, Bojórquez y Reyes (2020), es viable, dadas las propiedades de la tierra en esta localidad, para ayudar a obtener un adecuado comportamiento térmico al interior del espacio. La contribución de esta investigación se orienta al mejoramiento del hábitat al interior de las viviendas del siglo XX y XXI, especialmente en aquellas construcciones tradicionales y vernáculas de la localidad aquí estudiada.

\section{AGRADECIMIENTOS}

Gracias a la UAdeC US por permitir desarrollar el proyecto Estudio comparativo del comportamiento térmico del BTC, Block y módulo con tapa de huevo, y la colaboración de alumnos para la construcción de los módulos.

\section{REFERENCIAS BIBLIOGRÁFICAS}

ASTM (1995). Standard Practice for In-Situ Measurement of Heat Flux and Temperature on Building Envelope Components.

Calderón, F. (2019). Evaluación del mejoramiento del confort térmico con la incorporación de materiales sostenibles en viviendas en autoconstrucción en bosa, Bogotá, Colombia. Revista Hábitat Sustentable, 9(2), 30-41.

Comisión Nacional para el Conocimiento y Uso de la Biodiversidad, CONABIO. Portal de Geoinformación Sistema Nacional de Información sobre Biodiversidad 2020. Recuperado de http:// www.conabio.gob.mx/informacion/gis/layouts/clima1mgw
Costantini, A.B., Carro Pérez, M.E. y Francisca, F.M. (2016). Evaluación del comportamiento térmico de una edificación reemplazando el material de la envolvente por suelo cemento. Argentina de Energías Renovables y Medio Ambiente, 4, $05.43-$ 05.53.

Delfín, C. A., Gallina, S. A. y López, C. A. (2014). El hábitat: definición, dimensiones y escalas de evaluación para la fauna silvestre. En S. A. Gallina y C. A. López (Eds.), Manual de Técnicas para el estudio de la fauna (pp. 283-317). Recuperado de http:// www2.inecc.gob.mx/publicaciones2/libros/717/cap13.pdf

Espinoza, G., Cordero, D., Ruíz, A. y Roux, R. S. (2017). Análisis de aislamiento en tres sistemas de muro como envolvente alternativo. Revista Legado de Arquitectura y Diseño, (21), 119 - 133.

García, S., Kochova, L., Pugliese, G. y Sopoliga, P. (2010). Uso de la energía en los edificios. Manual para estudiantes. Proyecto IUSES y Programa de Energía Inteligente para Europa. Recuperado de https://docplayer.es/16550130-Uso-de-la-energia-en-losedificios-manual-para estudiantes.html

Herrera, L. C. (2017). Evaluación térmica del material isoblock en el clima cálido seco de cd. Juárez, México. Revista Hábitat Sustentable, 7(2), 18-27.

Huelsz, G., Molar, M. E. y Velázquez, J. (2014). Transferencia de calor en la envolvente arquitectónica y en el ser humano. En M. E. Molar, J. Velázquez y J. López, Vivienda 2 (pp. 7-22). Saltillo: Universidad Autónoma de Coahuila.

Luna L. A. (2019). CONFORT ANSI ASHRAE. Herramienta diseñada en Excel para estimar límite de confort. Mexicali: UABC.

Molar, M. E. y Huelsz, G. (2017). Comparación del comportamiento térmico de muros de concreto armado y de bloques de concreto huecos. Revista Legado de Arquitectura y Diseño, 1(22), 1-12.

Molar, M. E., Velázquez, J. y Gómez, A. B. (2018). Estudio del comportamiento térmico de las viviendas respecto a su orientación. México: Revista de Arquitectura y Diseño, 2(3), 1-7. Molar, M. E., Ríos, J., Bojórquez, G. y Reyes, J. A. (2020). Determinación de profundidad óptima para intercambiadores de calor tierra-aire en Saltillo, Coahuila. Research Journal Aplicaciones de la Ingeniería, 1(1), 1-15.

Molina, J. O., Lefebvre, G., Horn, M. y Gómez, M. M. (2020). Diseño de un módulo experimental bioclimático obtenido a partir del análisis de simulaciones térmicas para el centro poblado de Imata (4519 m s.n.m.) ubicado en Arequipa, Perú. Inf. tecnol., 31(2) 173 186. http://dx.doi.org/10.4067/S0718-07642020000200173.

Morris, G. D. (2017). Building Heat Transfer. Estados Unidos de América: Wiley.

Múnera, M. C. (2011). Construcción social del hábitat: Más allá de una política de producción de vivienda. Recuperado de http:// construccionsocialdelhabitat. files. wordpress. com/2011/04/ construccic3b3n-social-del-hc3a1bitat. pdf.

Muñoz Vásquez, M., Marino, B. M. y Thomas, L. P. (2015). Caracterización del comportamiento térmico de un edificio prototipo en Tandil (Buenos Aires). Revista ANALES AFA, 26(2), 1-10. Recuperado de https://afan.df.uba.ar/journal/index.php/ analesafa/article/view/2038 
Organización Panamericana de Salud (OPS). (octubre de 1999). Documento de Posición (OPS) sobre Políticas de Salud en la Vivienda. Organización Mundial de la Salud Organización Panamericana de la Salud. Recuperado de: http://www.bvsde. paho.org/bvsasv/e/iniciativa/posicion/posicion.html

Rodríguez, N. A., Nájera, M. y Martín, I. R. (2018). Análisis del desempeño térmico de los sistemas constructivos de un edificio de oficinas mediante simulaciones dinámicas. Revista Ingeniería Investigación y tecnología, XIX(3), 279-289. http://dx.doi. org/10.22201/fi.25940732e.2018.19n3.024

Roux, R. S. (2018). Bahareque y su inercia térmica para muros de vivienda. Revista LEGADO de Arquitectura y Diseño, (23), 25-32.

SENER y CONUEE (2011). Guía rápida para el cálculo de la NOM 020. Recuperado de https://energypedia.info/images/6/6d/GIZ_ Gu\%C3\%ADa_r\%C3\%A1 pida_NOM-020-ENER-2011.pdf

Szokolay, S. (2014). Introduction to architectural science: the basis of sustainable design. Third edition. London: Architectural Press.

Velázquez, J. y Molar, M. E. (2016). Material alternativo con tapas de huevo para cubierta. Revista del Desarrollo Urbano y Sustentable, 2(4), 35-42. 